\title{
Agricultores expuestos a compuestos organofosforados en el sitio la Cuca, cantón Arenillas, provincia de El Oro
}

Carmen Silverio; Geovanny Ramón; Elizabeth Guzmán¹ Universidad Técnica de Machala

csilverio@utmachala.edu.ec

\section{Resumen}

Conformando la provincia de El Oro se encuentra el cantón Arenillas en donde se ubica el sitio LA CUCA, que agrupa un gran número de agricultores dedicados a emplear sustancias organofosforadas. El efecto del uso de los compuestos organofosforados es inhibir la acetilcolinesterasa generando problemas a nivel respiratorio, dérmico e intestinal que incide en aproximadamente 300 trabajadores del sector. Medir los niveles de colinesterasa eritrocitaria en 70 agricultores como indicativo de intoxicación mediante método espectrofotométrico es de gran importancia para un diagnóstico de intoxicación; un estudio descriptivo logró determinar que entre 41 hombres y 4 mujeres, 20 de ellos es decir un 44,44\% fueron detectados con un valor de colinesterasa por debajo de lo normal. Concluyendo que la inhalación y manipulación de sustancias organofosforadas por un tiempo prolongado afectan la salud.

Palabras clave: Organofosforados, colinesterasa eritrocitaria, Método cinético

\section{Abstract}

La Cuca area which is located in Arenillas canton as a part of El Oro province pools together a great number of farmers who are dedicated to use organophosphates substances. The effect of the use of organophosphates compounds is to inhibit the acetylcholinesterase which causes problem in the respiratory tract, dermis and intestinal tract and it affects 300 workers of the area. It is very important to measure the level of cholinesterase erythrocyte in 70 farmers as an indicator of intoxication diagnostic through the spectrophotometric method; a descriptive study had identified that among 41 men and 4 women, 20 of them which represent the $45.65 \%$ were detected with a lower value of cholinesterase than normal.

In conclusion the prolonged inhalation and manipulation of organophosphate substances affects the health.

Keywords: Organophosphates, Erythrocyte Cholinesterase

Cómo citar este artículo: Silverio C., Ramón G. y Guzmán E. (2015) Agricultores expuestos a compuestos organofosforados en el sitio la Cuca, cantón Arenillas, provincia de El Oro. CUMBRES, Revista Científica. 1(2) 35 - 37 


\section{Introducción}

Según el Ministerio de Salud Pública (MSP) desde el año 2007 la causa principal de intoxicación es el uso de compuestos organofosforados que tienen un alto grado de toxicidad y ha aumentado un $24.4 \%$ anual en Ecuador.

En la Provincia de El Oro, cantón Arenillas, el sitio la Cuca es un sector agrícola dedicado al cultivo de arroz, lo que hace necesario el control de plagas por medio de sustancias químicas: insecticidas, herbicidas, acaricidas y fungicidas, esto junto al expendio no.

La escaza información por parte de las entidades de salud sobre efectos como (cáncer, leucemia y linfomas), que causan los agrotóxicos útiles en un 90\% a los agricultores es uno de los principales factores para el deterioro del organismo razón que nos lleva a investigar sobre el manejo de aquellos y sus consecuencias.

Controlado y el uso irracional de estas sustancias agravan el antes mencionado problema.

La composición de organofosforados y su función anticolinesterácicos derivados del ácido fosfórico fosforilan la enzima acetilcolinesterásica lo que implica la separación de la acetilcolina e impide la transmisión de impulsos nerviosos al cerebro provocando intoxicación y muerte. (Ruiz y Lozano).

Considerando de gran importancia conocer los agrotóxicos de mayor uso por parte de los agricultores de arroz de esta zona, y los niveles de inhibición de la enzima colinesterasa.

\section{Materiales y métodos}

Esta investigación es de tipo Descriptivo, mediante método cuali-cuantitativo.

Este estudio se basó en el método cinético con apoyo en la técnica de punción y manejo de muestras estandarizado por la Organización Mundial de la Salud (OMS).

Se contó con la colaboración de 70 trabajadores agrícolas expuestos a organofosforados y elegidos aleatoriamente de un grupo de 300, de quienes se obtuvo muestra de sangre y posteriormente con el uso de un anticoagulante se procedió a obtener el plasma.

Se utilizaron equipos de laboratorios como centrífuga, baño maría, espectrofotómetro, micropipetas, pipetas y cronómetro, al igual insumos de laboratorio clínico y reactivo (kit)

en la determinación de colinesterasa.

Para la obtención de resultados se incubó a 370C con valores de referencia de 4.970 y 13.997U/I para la aplicación de la fórmula.

El tipo de análisis estadístico fue descriptivo y sometido al análisis de los participantes mediante un documento firmado como consentimiento informado que fue aceptado por cada uno de ellos.

\section{Resultados}

La evaluación del trabajo de campo en LA CUCA, con respecto al uso de insecticidas, brinda información del nombre del agrotóxico, su principio activo y la categoría toxicológica.

Cuadro1a. Clasificación de los agrotóxicos por su aplicación.

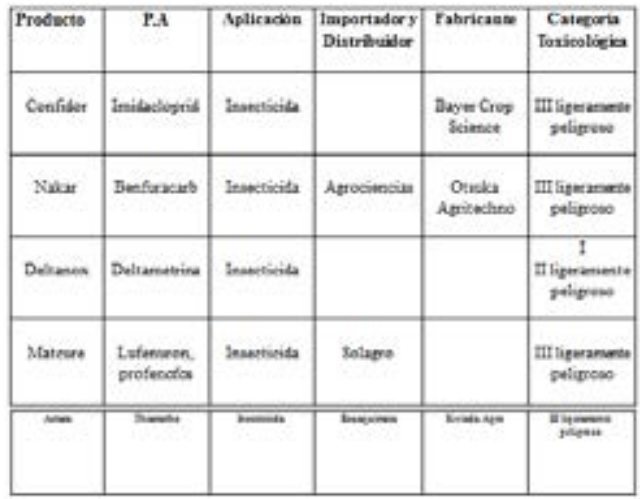

Cuadro1b. Clasificación de los agrotóxicos por su aplicación

\begin{tabular}{|c|c|c|c|c|c|}
\hline Crin & Fowan & macidis & Phatool & & 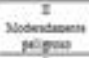 \\
\hline Nowar & Non: & buktude & akd & teis & and \\
\hline $2 \sec x$ & Phat & Lacids & ates & Bwininteind & 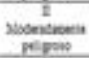 \\
\hline Sing & Sention & xumenos & ate & $\begin{array}{c}\text { Shes oned } \\
\text { inved }\end{array}$ & 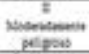 \\
\hline $\operatorname{Ten}$ & Shesisen & Bakiva & A tork & $x=2$ & $50=$ \\
\hline enos & Dentit. & mantion & स्रक्ष & finesciens & 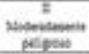 \\
\hline Shinve: & Wroet & luscioa & NWEK & $\begin{array}{l}\text { Sinoten } \\
\text { Sispolmo }\end{array}$ & BNines \\
\hline ont & Xeser: & Jukticas & Sostapo & Traution & Deneser \\
\hline रrues & 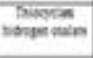 & Themena & Fatop & X्रmenans & Nosencent \\
\hline
\end{tabular}

\section{Cumbres}


En el cuadro de información agrotóxicos aplicados como insecticidas, obtenido como resultado de investigación de campo en el estudio, se resalta la presencia de 4 agrotóxicos considerados altamente peligrosos por su composición química, de uso regular por los agricultores en fumigaciones.

Cuadro1c. Clasificación de los agrotóxicos por su aplicación

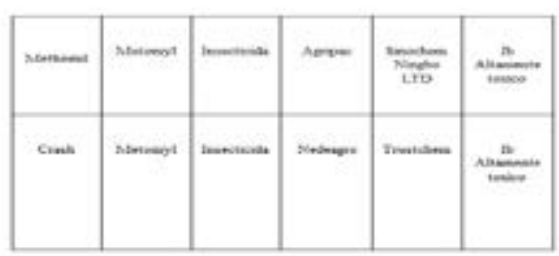

Considerando la población estudiada en el sitio la Cuca, se ha determinado mediante encuestas el porcentaje de población por sexo.
El resultado obtenido sobre el tiempo de exposición de los agricultores a los agrotóxicos de alta y ligera toxicidad, a partir de los 5 años consecutivos de labores hata los 53 años de edad se evidencia e la siguiente tabla .

En resumen los datos descritos, indican que los agricultores expuestos a agrotóxicos regularmente a partir de 26 a 30 años de labores poseen un mayor porcentaje de afectación. En adelante esto disminuye, debido a la deserción de los trabajadores en la actividad agrícola por presentar problemas en su salud, disminuyendo el número de trabajadores dedicados a esta actividad.

El criterio para la selección de insecticidas de uso doméstico intra domiciliario debe contemplarse en la ley orgánica de salud en función de la naturaleza química del producto y su grado de toxicidad.
Cuadro1c. Clasificación de los agrotóxicos por su aplicación

\begin{tabular}{|c|c|c|}
\hline Variable & Frecuencia & $\%$ \\
\hline Masculino & 41 & 91 \\
\hline Femerino & 4 & 9 \\
\hline
\end{tabular}

EXPOSICIÓNA ORGANOFOSFORADOS

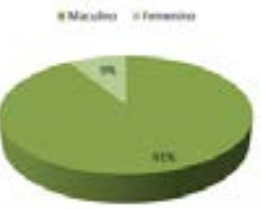

Tabla 1. Años de exposición laboral a insecticidas.

\begin{tabular}{|c|c|}
\hline Años de exposicion & \%Población affectada \\
\hline 5-10ANOS & $2273 \%$ \\
\hline 11-15 ANIOS & $6.81 \%$ \\
\hline 20.25 & $9.09 \%$ \\
\hline $26 \cdot 30$ & $1363 \%$ \\
\hline $31-36$ & $2273 \%$ \\
\hline $37-41$ & $2273 \%$ \\
\hline 42.47 & $2273 \%$ \\
\hline 48.53 & 0 \\
\hline
\end{tabular}

\section{Referencias bibliográficas}

- COLLADO, A. 82001). Intoxicación por insecticidas Órganos fosforados, Granada, España.

- HARRINSON. (1998). Principios de, Medicina Interna. México.
- MARTíNEZ, P. (2005). Etiología, procedimientos y Diagnóstico. Madrid, España.

- CARMONA, F. (2003). Valores de eferencia de colinesterasa plasmática con los métodos de Michel, EQM y Monotest en población laboral activa del departamento de Antioquia, Colombia. 